\title{
Review Article Effects of Qigong on Depression: A Systemic Review
}

\author{
Byeongsang Oh, ${ }^{1,2}$ Sun Mi Choi, ${ }^{3}$ Aya Inamori, ${ }^{4}$ David Rosenthal, ${ }^{1}$ and Albert Yeung ${ }^{4}$ \\ ${ }^{1}$ Dana-Farber Cancer Institute and Harvard Medical School, 450 Brookline Avenue, Boston, MA 02215, USA \\ ${ }^{2}$ Sydney Medical School, University of Sydney, Edward Ford Building A27, Fisher Road, Camperdown, NSW 2006, Australia \\ ${ }^{3}$ Korea Institute of Oriental Medicine, Acupuncture, Moxibustion \& Meridian Research Centre, 1672 Yuseongdae-ro, \\ Yuseong-gu, Daejeon 305-811, Republic of Korea \\ ${ }^{4}$ Massachusetts General Hospital and Harvard Medical School, 55 Fruit Street, Boston, MA 02114, USA \\ Correspondence should be addressed to Byeongsang Oh; boh1@partners.org
}

Received 19 November 2012; Revised 25 January 2013; Accepted 29 January 2013

Academic Editor: Ching Lan

Copyright (C) 2013 Byeongsang Oh et al. This is an open access article distributed under the Creative Commons Attribution License, which permits unrestricted use, distribution, and reproduction in any medium, provided the original work is properly cited.

\begin{abstract}
Physical exercises and relaxation have been found to be beneficial for depression. However, there is little evidence on the use of Qigong, a mind-body practice integrating gentle exercise and relaxation, in the management of depression. The aim of this paper is to evaluate the effects of Qigong on depression. The paper examined clinical trials measuring the effect of Qigong on depression within six large-scale medical research databases (PubMed, Medline, ProQuest, Science Direct, EMBASE, and PsycInfo) till October 2011. Key words "Qigong," "depression," and "mood" were used. Ten studies were identified as original randomized controlled trial (RCT) studies investigating the effect of Qigong on depression as primary $(n=2)$ or secondary outcome $(n=8)$. Four studies reported positive results of the Qigong treatment on depression; two reported that Qigong effect on depression was as effective as physical exercise. One study reported that Qigong was comparable to a conventional rehabilitation program, but the remaining three studies found no benefits of Qigong on depression. While the evidence suggests the potential effects of Qigong in the treatment of depression, the review of the literature shows inconclusive results. Further research using rigorous study designs is necessary to investigate the effectiveness of Qigong in depression.
\end{abstract}

\section{Introduction}

Depression is a common illness occurring in approximately $5-13 \%$ of women and $2-8 \%$ of men at any particular point in time. Depression has a lifetime prevalence rate of $16.2 \%$ and a twelve-month prevalence rate of $6.6 \%[1,2]$. By 2020 , depression is projected to become the second global leading cause of disability [3]. The annual cost of depression in the United States exceeds $\$ 80$ billion, surpassing that of other chronic illnesses such as diabetes and hypertension [4]. Depression not only accounts for up to $70 \%$ of psychiatric hospitalizations and $60 \%$ of suicides, but also complicates the management of other diseases [5]. Despite the growing number of marketed antidepressants, between 19 and 34\% of patients with depression do not respond to acute antidepressant treatment. $29-46 \%$ of patients with depression fail to achieve full remission, and up to $50 \%$ of patients experience recurrence [6,7]. Given the scale of this problem, there is a need to explore alternative and complementary forms of treatment. In recent years, there has been a growing interest in alternative medical approaches to treating depression, including acupuncture $[8,9]$, Tai Chi $[10]$, meditation $[11,12]$, and Qigong [13, 14].

Qigong is a traditional Chinese mind-body medicine dating back to over one thousand years. It consists of two types: internal and external Qigong. Internal Qigong is a form of mind-body medicine that involves coordinated gentle exercise and relaxation through meditation and breathing [15]. The practice of internal Qigong promotes balance and is believed to combat energy blockages by facilitating the flow of vital energy around the body [16]. In doing so, it contributes to both physical and psychological well-being. External Qigong, on the other hand, is a branch of energy medicine in which an experienced Qigong practitioner sends or emits Qi-a form of energy - to a patient for the treatment of that patient's illnesses [17].

Existing literature has reported that internal Qigong decreases heart rate [18], blood pressure [19], lipid levels [19], 
and circulating stress hormones [20] as well as improves the body's immune function [20, 21]. Moreover, a recent review which examined 77 articles on the physiological and psychological effects of Tai Chi and Qigong suggests that both Tai Chi and Qigong have beneficial effects on bone density, cardiopulmonary functions, physical and immune functions, self-efficacy, and quality of life and improve psychological symptoms [22]. Most of the earlier studies on Qigong recruited subjects with a variety of medical conditions; however, only a few specifically examined the effects of Qigong on patients with depression. This paper aims to fill the gap in the literature by examining the reported effects of internal Qigong on depression and demonstrates the need for further research.

\section{Methods}

A literature search reviewing all published articles prior to October 2011 on the effect of Qigong on depression was conducted using PubMed, Medline (1950 ), ProQuest (1950 ), ScienceDirect (1950 ), EMBASE, and PsycINFO (1806 ). Key words "Qigong," "depression," and "mood" were used in the literature search. Identified records were initially screened for eligibility based on title and abstract. Reference lists of identified papers and reviews were manually searched for additional studies in related areas. Articles were finally selected based on the analysis of the full text. Two reviewers, B. Oh, and A. Yeung, independently applied the inclusion criteria. The two reviewers compared results and resolved any discrepancies by agreement.

\section{Eligibility Criteria}

Studies which investigated the effects of Qigong on depression as primary or secondary outcomes based on randomized controlled trial (RCT) design were eligible. Articles reporting on interventions using external Qigong or Tai Chi were excluded. The literature search included only papers with the full article published in English.

\section{Results}

The initial literature search identified 520 articles using the key words "Qigong," "depression," and "mood," of which 425 non-Qigong clinical trial articles were excluded. Of the 95 remaining articles, 10 articles met the eligibility criteria and were reviewed, as shown in Figure 1. Of the ten studies shown in Table 1, two studies measured depression as a primary outcome $[13,23]$ and eight studies measured depression as a secondary outcome. The former two studies measured the effects of Qigong on geriatric depression.

In one of these two studies, Tsang et al. [23] showed that Qigong was as effective as a conventional rehabilitation program in a pilot study. In a subsequent study with a larger sample size, Tsang et al. [13] demonstrated a significant difference between the Qigong intervention and the control group. The remaining eight studies measured depression as a secondary outcome among patients who were recruited

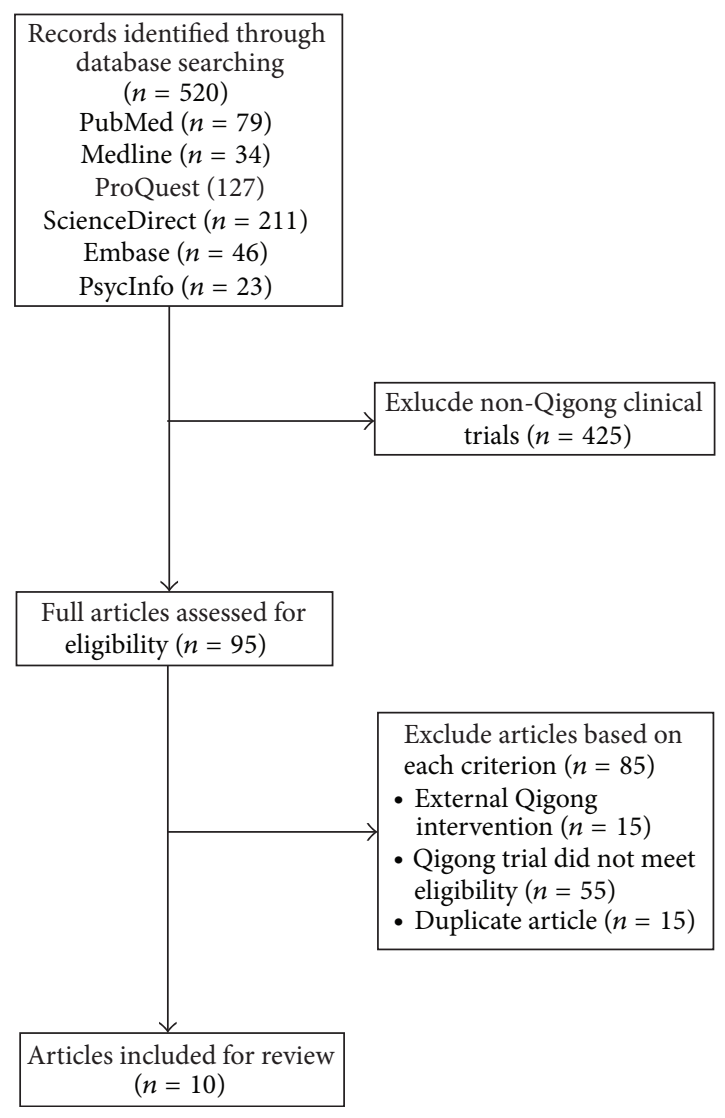

Figure 1: Flowchart of review process.

based on other medical conditions. Of eight studies, three studies reported a significant difference between Qigong intervention and control groups [24-26]. Two studies showed that Qigong was as effective as physical exercise [27, 28]. Three studies did not find a significant difference between the Qigong intervention and the control group [29-31].

These studies were conducted in Hong Kong $(n=3)$, Korea $(n=1)$, Germany $(n=2)$, Sweden $(n=2)$, Australia $(n=1)$, and the USA $(n=1)$ between 2003 and 2009 . Among them, there were ten RCTs, which employed two arms (Qigong versus control group). Six of these ten studies used a sham intervention for the control groups. None of the studies used a double-blind design.

Sample sizes ranged from 50 to 162 subjects. Study populations varied, with two studies conducted on geriatric patients with depression. Other study populations included patients with fibromyalgia, hypertension, Parkinson's disease, cancer, burnout, severe chronic pain, and female college students. One study did not report a detailed demographic profile of its participants, as the study was conducted during a Qigong retreat [32]. The mean age of participants ranged from 19 to 82 .

The duration of Qigong interventions also varied across studies. Qigong intervention period ranged from 6 to 16 weeks ( 6 weeks $(n=1), 8$ weeks $(n=2), 12$ weeks $(n=4)$, and 16 weeks $(n=2))$, and one study involved a four-day Qigong retreat. The duration of each intervention also varied 


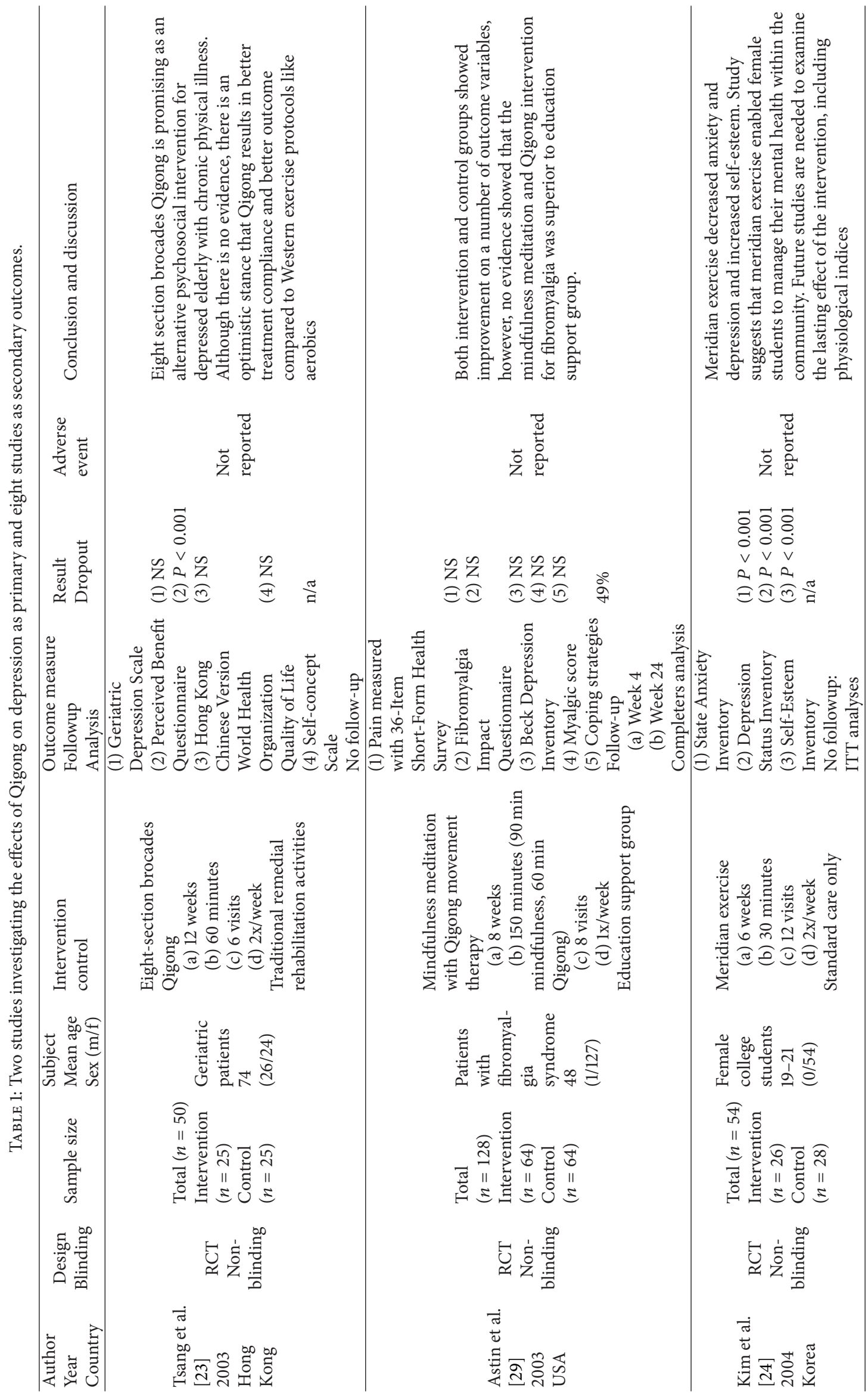




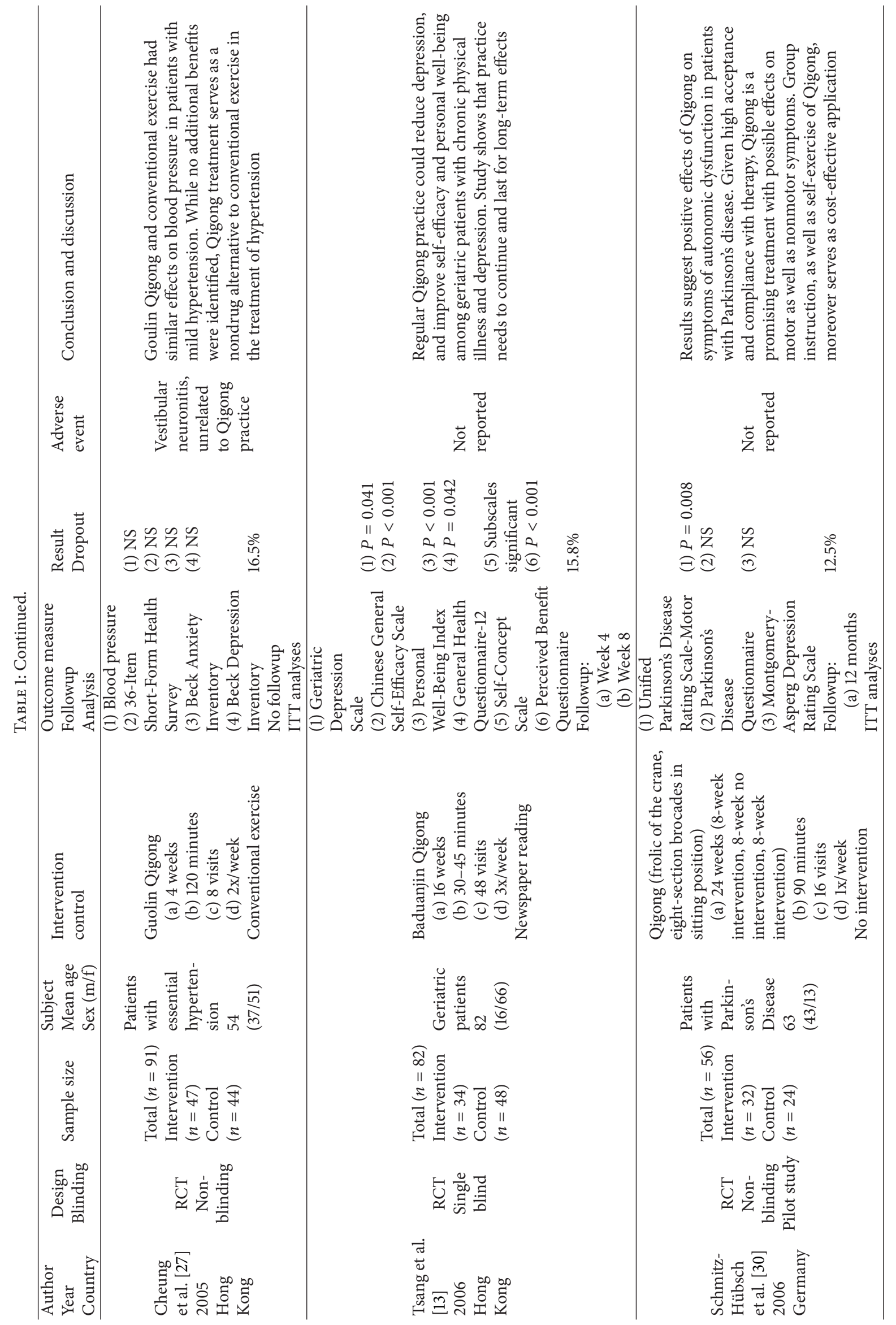




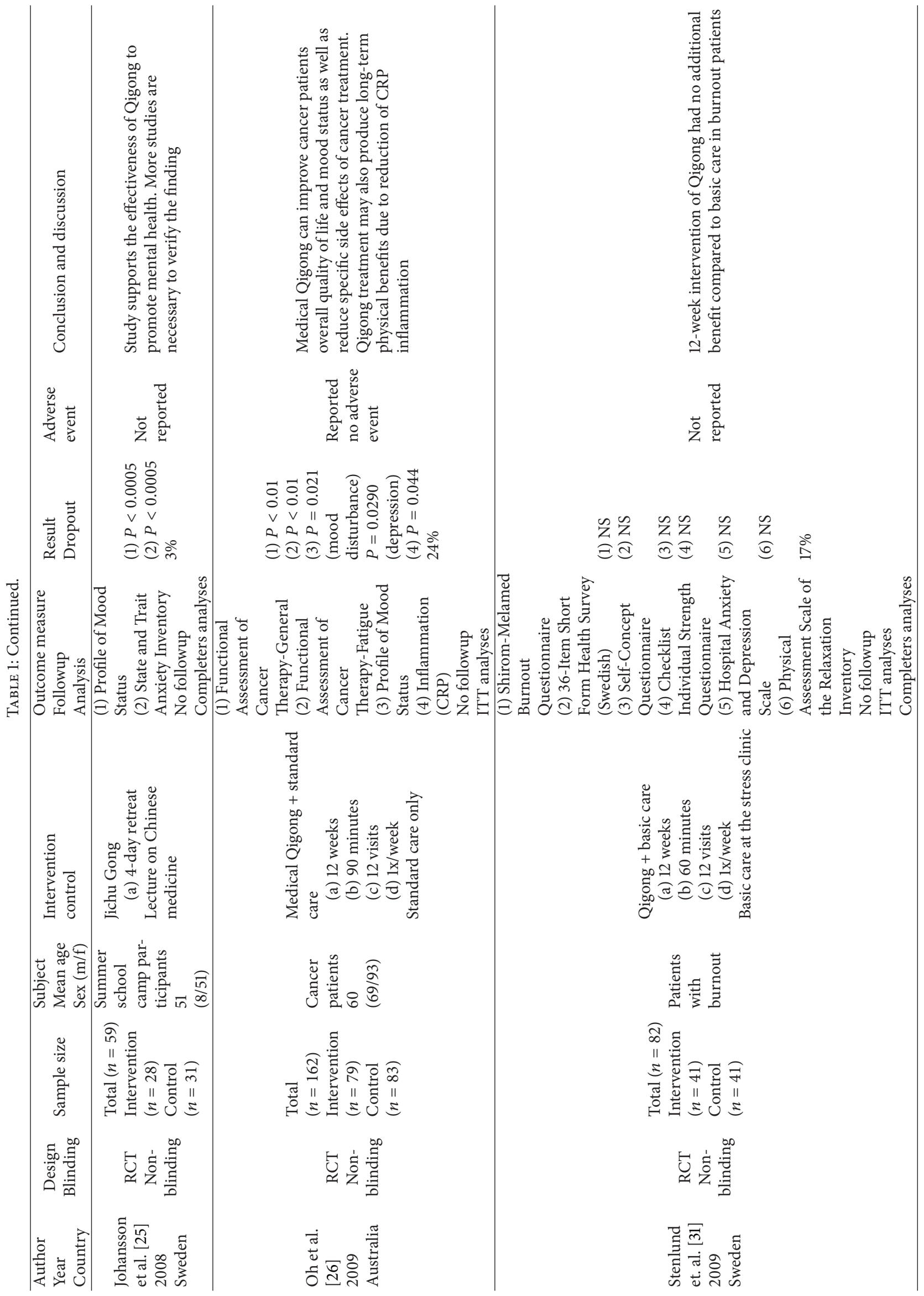


from 30 minutes $(n=1), 45$ minutes $(n=2), 60$ minutes $(n=3)$, and 90 minutes $(n=2)$ to 120 minutes $(n=1)$. Most studies $(n=7)$ offered Qigong interventions twice a week. One study [32] did not describe the length or frequency of the intervention. Three out of the 10 studies had long-term follow-up assessments at 24 weeks [29], 6 months [28], and 12 months [30].

The ten studies used different instruments to measure depression outcomes. Two studies used the Geriatric Depression Scale (GDS), two used the Beck Depression Inventory (BDI), and two used the Profile of Mood States (POMS). Other instruments included the Depression Status Inventory (DSI), Montgomery-Asberg Depression Rating Scale (MADRS), Hospital Anxiety and Depression Scale (HADS), and Allgemeine Depressionsskala (ADS).

With the exception of one study that had an unusually high dropout rate of $49 \%$ [29], most studies had satisfactory dropout rates ranging from $3 \%$ to $24 \%$. Two studies did not report their dropout rates [23, 24]. Two studies [27, 28] reported adverse events that were not directly related to the practice of Qigong; eight studies did not report adverse events; and one reported no adverse effects of Qigong intervention [26]. Two studies which recruited patients with hypertension and chronic neck pain reported adverse events not specifically related to the intervention, such as muscle ache, tension, nausea, and vestibular neuronitis.

Of the ten studies, five conducted intention-to-treat (ITT) statistical analyses, two conducted completers analyses [25, 29], and one performed both ITT and completer analyses [31]. Two studies did not report the details of their data analysis methods $[13,23]$.

\section{Discussion}

This paper suggests that the effect of Qigong in the treatment of depression is inconclusive, although potential effects of Qigong in the treatment of depression was supported by the biopsychosocial model [33], relaxation response theory [34, $35]$, and evidence on the positive effects of exercise $[36,37]$. Further, our review result was not consistent with the previous review conducted by Jahnke et al., which reported the effect of Qigong and Tai Chi in the treatment of psychological symptoms including depression [22]. Their review concluded that Qigong and Tai Chi can reduce psychological symptoms including depression. The differences in results may be due to the different inclusion criteria used in the two reviews. In Jahnke et al.s review [22], their results were based more on tai chi studies than on Qigong studies, while our review assessed exclusively Qigong studies. Although, tai chi is considered as part of moving Qigong, the differences and similarities of basic philosophy and practices between tai chi and Qigong are debated among the academic researchers, particularly in Western world $[22,38]$. Interestingly, the result of this review is similar to a recent review conducted by Tsang et al. with 12 RCTs which compared the effects of mindful exercises versus nonmindful exercises [39]. This review reported that both mindful and nonmindful physical exercises were effective in the treatment of depression or depressive symptoms in the short term. Our paper also showed that Qigong was as effective as physical exercise and rehabilitation program for treatment of depression. Results from Jahnke et al. [22] and our review are compatible with two recent reviews on the efficacy of exercise on depression, both concluding that exercise has a mild treatment effect on depression [36, 37]. These findings suggest that future studies are needed to examine the mechanism of the effects of Qigong, tai chi, and physical exercise on the brain to decipher the similarities and differences of their effects on depression.

One of most remarkable findings of this review $(n=10)$ was that participants did not report any psychotic reactions from Qigong, as previous literature has indicated as possible concern [25]. The inconsistent results based on the above reviews may reflect dose response of subjects receiving Qigong intervention with different frequencies, lengths, and intensities. A limitation of this review is that it included studies with small sample sizes and no appropriate sham intervention for control group, no blinding of subjects and Qigong instructors, and they used various instruments to measure depression outcomes. There was moreover a publication selection bias, as we only examined studies published in English.

Future studies may take into account the following suggestions for methodology. First, participants who meet the criteria for major depressive disorder, dysthymic disorder, or depressive disorder not otherwise specified based on DSMIV criteria should be included. Since Qigong originated in the East, different ethnic groups may be recruited to examine if cultural differences could be a mediator or moderator of treatment outcomes. A three-arm design (Qigong intervention versus sham Qigong versus usual care or waitlist group) with adequate sample size is recommended to detect statistical and clinical significance, as suggested by $\mathrm{Oh}$ et al. [26]. Dose-response relationship can be examined by varying length (e.g., 30 minutes versus 60 minutes versus 90 minutes), frequency (e.g., weekly versus biweekly versus every 4 weeks), and intensity of intervention, as measured by physical activity intensity scale. Both quantitative and qualitative outcome measures are recommended in order to capture the complexity of depression treatment effect. Measureing of biomarkers, such as immune function, cytokines, and DNA damage level, may provide objective information on the physiological and psychological effects of Qigong intervention. Finally, a cost-benefit analysis could examine possible health policy considerations.

In conclusion, all studies suggest that Qigong intervention for patients with major depressive disorder is safe and feasible; however, evidence for its effectiveness is limited. Future study with more robust design is warranted.

\section{Conflict of Interests}

B. Oh, S. M. Choi, A. Inamori, D. Rosenthal, and A. Yeung, confirm that there are no known conflict of interests associated with this publication and there has been no significant financial support for this work that could have influenced its outcome. 


\section{Acknowledgment}

This work was supported by the Development of Acupuncture, Moxibustion and Meridian Standard Health Technology (K13010), Korea Institute of Oriental Medicine (KIOM).

\section{References}

[1] H. A. Pincus and A. R. Pettit, "The societal costs of chronic major depression," Journal of Clinical Psychiatry, vol. 62, supplement 6, pp. 5-9, 2001.

[2] R. C. Kessler, P. Berglund, O. Demler et al., “The epidemiology of major depressive disorder: results from the National Comorbidity Survey Replication (NCS-R)," Journal of the American Medical Association, vol. 289, no. 23, pp. 3095-3105, 2003.

[3] C. D. Mathers and D. Loncar, "Projections of global mortality and burden of disease from 2002 to 2030," PLoS Medicine, vol. 3, no. 11, article e442, 2006.

[4] R. DeVol and A. Bedroussian, An Unhealthy America: The Economic Burden of Chronic Disease Charting a New Course to Save Lives and Increase Productivity and Economic Growth, Milken Institute, Santa Monica, Calif, USA, 2007.

[5] C. A. Mancuso, M. G. E. Peterson, and M. E. Charlson, "Effects of depressive symptoms on health-related quality of life in asthma patients," Journal of General Internal Medicine, vol. 15, no. 5, pp. 301-310, 2000.

[6] M. Fava and K. G. Davidson, "Definition and epidemiology of treatment-resistant depression," Psychiatric Clinics of North America, vol. 19, no. 2, pp. 179-200, 1996.

[7] M. Fava, "New approaches to the treatment of refractory depression," Journal of Clinical Psychiatry, vol. 61, supplement 1, pp. 26-32, 2000.

[8] D. Mischoulon, C. D. Brill, V. E. Ameral, M. Fava, and A. S. Yeung, "A pilot study of acupuncture monotherapy in patients with major depressive disorder," Journal of Affective Disorders, vol. 141, no. 2-3, pp. 469-473, 2012.

[9] C. Andreescu, R. M. Glick, C. A. Emeremni, P. R. Houck, and B. H. Mulsant, "Acupuncture for the treatment of major depressive disorder: a randomized controlled trial," Journal of Clinical Psychiatry, vol. 72, no. 8, pp. 1129-1135, 2011.

[10] C. Wang, R. Bannuru, J. Ramel, B. Kupelnick, T. Scott, and C. H. Schmid, "Tai Chi on psychological well-being: systematic review and meta-analysis," BMC Complementary and Alternative Medicine, vol. 10, article 23, 2010.

[11] G. R. Sharplin, S. B. Jones, B. Hancock, V. E. Knott, J. A. Bowden, and H. S. Whitford, "Mindfulness-based cognitive therapy: an efficacious community-based group intervention for depression and anxiety in a sample of cancer patients," The Medical Journal of Australia, vol. 193, no. 5, supplement, pp. S79-S82, 2010.

[12] C. J. Hoffman, S. J. Ersser, J. B. Hopkinson, P. G. Nicholls, J. E. Harrington, and P. W. Thomas, "Effectiveness of mindfulnessbased stress reduction in mood, breast- and endocrine-related quality of life, and well-being in stage 0 to III breast cancer: a randomized, controlled trial," Journal of Clinical Oncology, vol. 30, no. 12, pp. 1335-1342, 2012.

[13] H. W. H. Tsang, K. M. T. Fung, A. S. M. Chan, G. Lee, and F. Chan, "Effect of a Qigong exercise programme on elderly with depression," International Journal of Geriatric Psychiatry, vol. 21, no. 9, pp. 890-897, 2006.

[14] H. W. H. Tsang, L. Cheung, and D. C. C. Lak, "Qigong as a psychosocial intervention for depressed elderly with chronic physical illnesses," International Journal of Geriatric Psychiatry, vol. 17, no. 12, pp. 1146-1154, 2002.

[15] J. Zhang, Qigong Exercise Therapy, Shandong Science and Technology Press, Beijing, China, 1997.

[16] J. A. Johnson, J. M. Stewart, and M. H. Howell, Chinese Medical Qigong Therapy: A Comprehensive Clinical Guide, International Institute of Medical Qigong, Pacific Grove, Calif, USA, 2000.

[17] M. S. Lee, M. K. Kim, and Y. H. Lee, "Effects of Qi-therapy (external Qigong) on cardiac autonomic tone: a randomized placebo controlled study," International Journal of Neuroscience, vol. 115, no. 9, pp. 1345-1350, 2005.

[18] M. S. Lee, M. S. Lee, E. S. Choi, and H. T. Chung, "Effects of Qigong on blood pressure, blood pressure determinants and ventilatory function in middle-aged patients with essential hypertension," American Journal of Chinese Medicine, vol. 31, no. 3, pp. 489-497, 2003.

[19] M. S. Lee, M. S. Lee, H. J. Kim, and E. S. Choi, "Effects of Qigong on blood pressure, high-density lipoprotein cholesterol and other lipid levels in essential hypertension patients," International Journal of Neuroscience, vol. 114, no. 7, pp. 777-786, 2004.

[20] H. Ryu, H. S. Lee, Y. S. Shin et al., "Acute effect of Qigong training on stress hormonal levels in man," American Journal of Chinese Medicine, vol. 24, no. 2, pp. 193-198, 1996.

[21] J. M. Manzaneque, F. M. Vera, E. F. Maldonado et al., "Assessment of immunological parameters following a Qigong training program," Medical Science Monitor, vol. 10, no. 6, pp. CR264CR270, 2004.

[22] R. Jahnke, L. Larkey, C. Rogers, J. Etnier, and F. Lin, "A comprehensive review of health benefits of Qigong and Tai Chi," American Journal of Health Promotion, vol. 24, no. 6, pp. el-e25, 2010.

[23] H. W. H. Tsang, C. K. Mok, Y. T. Au Yeung, and S. Y. C. Chan, "The effect of Qigong on general and psychosocial health of elderly with chronic physical illnesses: a randomized clinical trial," International Journal of Geriatric Psychiatry, vol. 18, no. 5, pp. 441-449, 2003.

[24] K. B. Kim, S. M. Cohen, H. K. Oh, and S. R. Sok, "The effects of meridian exercise on anxiety, depression, and self-esteem of female college students in Korea," Holistic Nursing Practice, vol. 18, no. 5, pp. 230-234, 2004.

[25] M. Johansson, P. Hassmén, and J. Jouper, "Acute effects of Qigong exercise on mood and anxiety," International Journal of Stress Management, vol. 15, no. 2, pp. 199-207, 2008.

[26] B. Oh, P. Butow, B. Mullan et al., "Impact of medical Qigong on quality of life, fatigue, mood and inflammation in cancer patients: a randomized controlled trial," Annals of Oncology, vol. 21, no. 3, article mdp479, pp. 608-614, 2009.

[27] B. M. Y. Cheung, J. L. F. Lo, D. Y. T. Fong et al., "Randomised controlled trial of Qigong in the treatment of mild essential hypertension," Journal of Human Hypertension, vol. 19, no. 9, pp. 697-704, 2005.

[28] P. von Trott, A. M. Wiedemann, R. Lüdtke, A. Reißhauer, S. N. Willich, and C. M. Witt, "Qigong and exercise therapy for elderly patients with chronic neck pain (QIBANE): a randomized controlled study," Journal of Pain, vol. 10, no. 5, pp. 501-508, 2009.

[29] J. A. Astin, B. M. Berman, B. Bausell, W. L. Lee, M. Hochberg, and K. L. Forys, "The efficacy of mindfulness meditation plus Qigong movement therapy in the treatment of fibromyalgia: a randomized controlled trial," Journal of Rheumatology, vol. 30, no. 10, pp. 2257-2262, 2003. 
[30] T. Schmitz-Hübsch, D. Pyfer, K. Kielwein, R. Fimmers, T. Klockgether, and U. Wüllner, "Qigong exercise for the symptoms of Parkinson's disease: a randomized, controlled pilot study," Movement Disorders, vol. 21, no. 4, pp. 543-548, 2006.

[31] T. Stenlund, L. S. Birgander, B. Lindahl, L. Nilsson, and C. Ahlgren, "Effects of Qigong in patients with burnout: a randomized controlled trial," Journal of Rehabilitation Medicine, vol. 41, no. 9, pp. 761-767, 2009.

[32] M. Johansson and P. Hassmén, "Acute psychological responses to Qigong exercise of varying durations," American Journal of Chinese Medicine, vol. 36, no. 3, pp. 449-458, 2008.

[33] Y. W. Y. Chow and H. W. H. Tsang, "Biopsychosocial effects of Qigong as a mindful exercise for people with anxiety disorders: a speculative review," Journal of Alternative and Complementary Medicine, vol. 13, no. 8, pp. 831-839, 2007.

[34] T. M. Edenfield and S. A. Saeed, "An update on mindfulness meditation as a self-help treatment for anxiety and depression," Psychology Research and Behavior Management, vol. 5, pp. 131141, 2012.

[35] H. Benson, The Relaxation Response, Morrow, New York, NY, USA, 1983.

[36] J. Krogh, M. Nordentoft, J. A. C. Sterne, and D. A. Lawlor, "The effect of exercise in clinically depressed adults: systematic review and meta-analysis of randomized controlled trials," Journal of Clinical Psychiatry, vol. 72, no. 4, pp. 529-538, 2011.

[37] G. E. Mead, W. Morley, P. Campbell, C. A. Greig, M. McMurdo, and D. A. Lawlor, "Exercise for depression," Cochrane Database of Systematic Reviews, no. 4, Article ID CD004366, 2008.

[38] L. Larkey, L. Szalacha, C. Rogers, R. Jahnke, and B. Ainsworth, "Measurement pilot study of the Meditative Movement Inventory (MMI)," Journal of Nursing Measurement, vol. 20, no. 3, pp. 230-243, 2012.

[39] H. W. H. Tsang, E. P. Chan, and W. M. Cheung, "Effects of mindful and non-mindful exercises on people with depression: a systematic review," British Journal of Clinical Psychology, vol. 47, no. 3, pp. 303-322, 2008. 


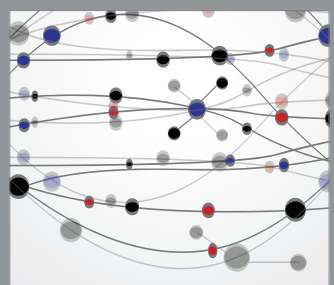

The Scientific World Journal
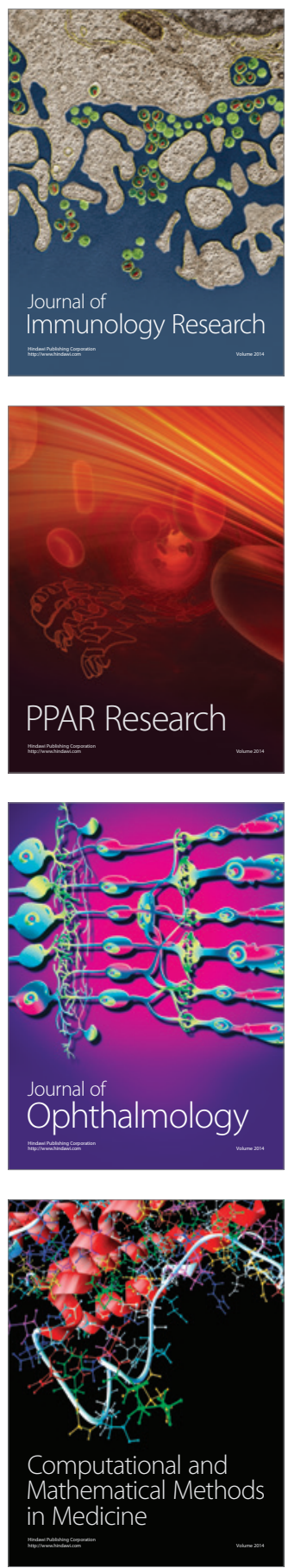

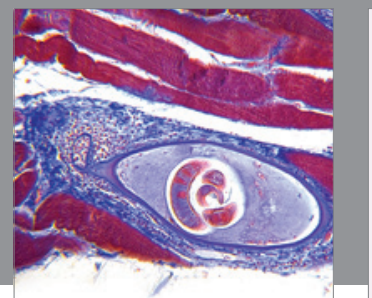

Gastroenterology

Research and Practice
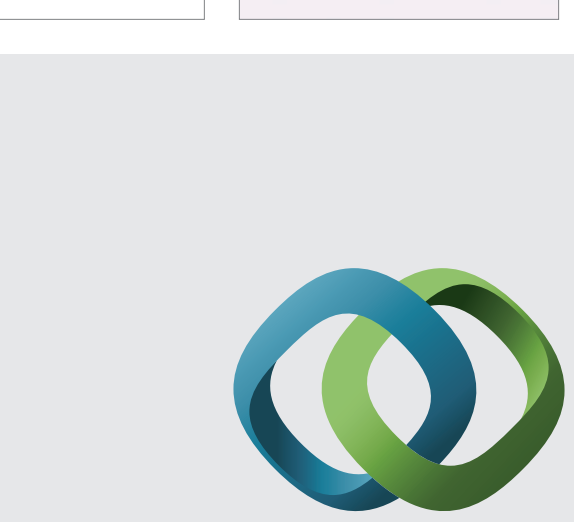

\section{Hindawi}

Submit your manuscripts at

http://www.hindawi.com
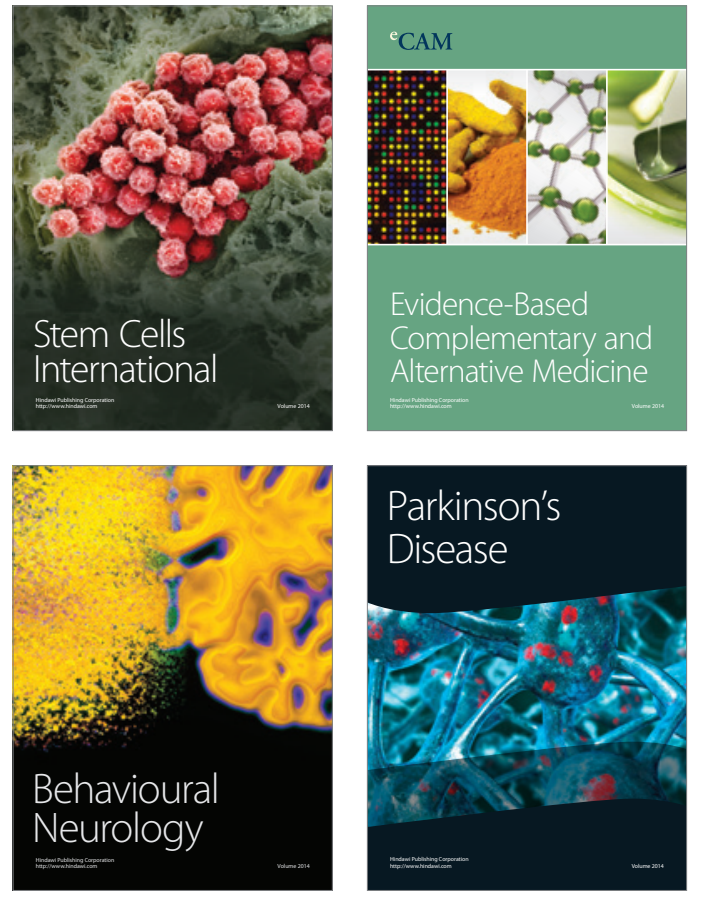
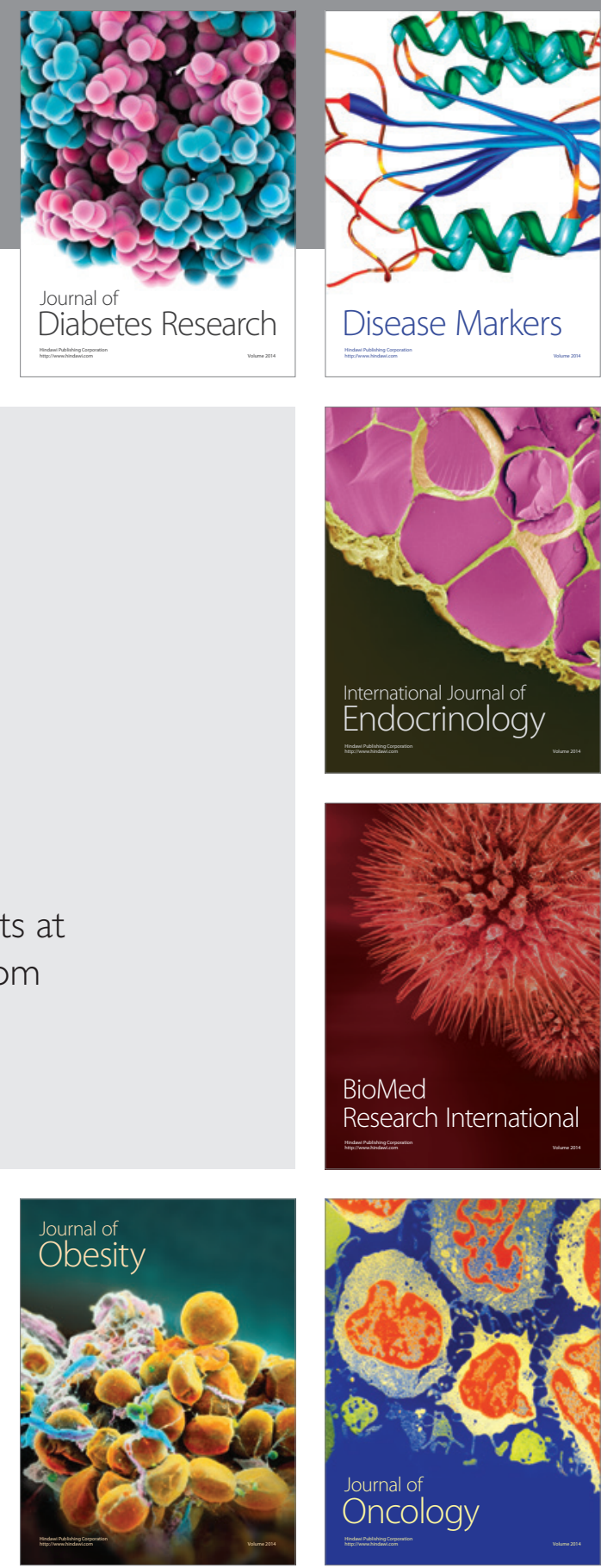

Disease Markers
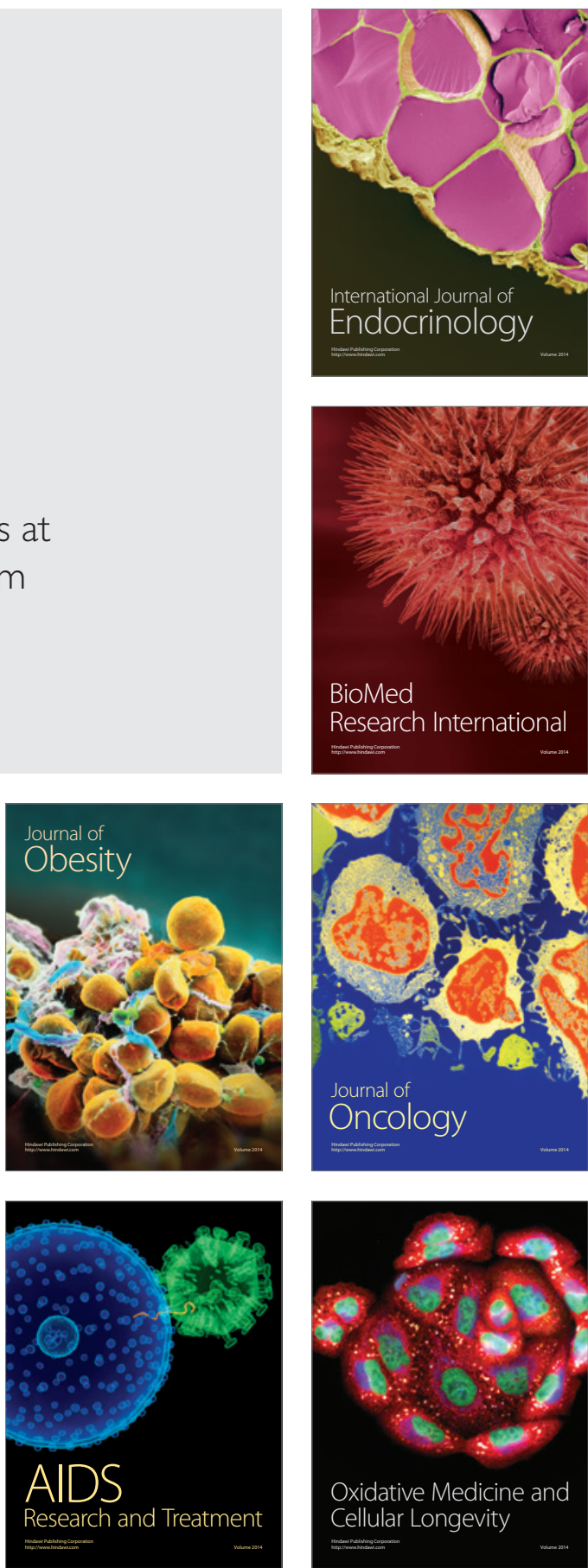